\title{
Transcriptome and Metabolome Analyses Reveal an Insight of Chlorophyll, Photosynthesis, Metal lon and Phenylpropanoids Related Pathways in Sugarcane Ratoon Chlorosis
}

Ting Luo

Guangxi Academy of Agricultural Sciences

\section{Zhongfeng Zhou}

Guangxi Academy of Agricultural Sciences

\section{Yuchi Deng}

Guangxi Academy of Agricultural Sciences

\section{Yegeng Fan}

Guangxi Academy of Agricultural Sciences

\section{Lihang Qiu}

Guangxi Academy of Agricultural Sciences

\section{Rongfa Chen}

Guangxi Academy of Agricultural Sciences

Haifeng Yan

Guangxi Academy of Agricultural Sciences

Huiwen Zhou

Guangxi Academy of Agricultural Sciences

Jianming Wu ( $\nabla$ wujianming2004@126.com )

Guangxi Academy of Agricultural Sciences

\section{Qi Chen}

New Technology Enterpreneur Center, Nanning

\section{Research Article}

Keywords: Ratoon sugarcane chlorosis, chlorophyll metabolism, photosynthesis, metal ion metabolism, phenylpropanoids biosynthesis

Posted Date: December 28th, 2021

DOI: https://doi.org/10.21203/rs.3.rs-1194932/v1

License: (a) (1) This work is licensed under a Creative Commons Attribution 4.0 International License. Read Full License 


\section{Abstract}

\section{Background}

Ratoon sugarcane (Saccharum officinarum) is susceptible to chlorosis, significantly reducing production. The molecular mechanism underlying this phenomenon remains unknown. We analyzed the transcriptome and metabolome of chlorotic and non-chlorotic sugarcane leaves from the same field to gain insight into the symptom.

\section{Results}

The agronomic traits, like plant height, leaf number, stalk nod number, and tiller number, declined in chlorotic sugarcane. The chlorophyll content in chlorosis leaves was significantly lower than non-chlorotic leaves. A total of 11,776 differentially expressed genes (DEGs) were discovered in transcriptome analysis. In the KEGG enriched chlorophyll metabolism pathway, sixteen DEGs were found, eleven of which were down-regulated. Two photosynthesis pathways were also enriched, with 32 genes downregulated and four genes upregulated. Among the 81 enriched $\mathrm{GO}$ biological processes, there were four categories related to metal ion homeostasis and three related to metal ion transport. Approximately 400 metabolites were identified in metabolome analysis. The thirteen classified differentially expressed metabolites (DEMs) were found all down-regulated. The phenylpropanoid biosynthesis pathway was enriched in DEGs and DEMs, indicating phenylpropanoids' vital role in chlorosis.

\section{Conclusions}

According to our study, chlorophyll production, metal ion metabolism, photosynthesis, and some secondary metabolites of the phenylpropanoid biosynthesis pathway, were considerably altered in chlorotic ratoon sugarcane. Our finding revealed the relation between chlorosis and these pathways, which would further the understanding of the mechanism of ratoon sugarcane chlorosis.

\section{Introduction}

Sugarcane (Saccharum officinarum) is an essential crop in economic and food safety. In China, sugarcane accounts for $90 \%$ of sugar production, contributing 6 to 8 billion RMB [1]. In Guangxi, the leading sugarproducing province in China, ratoon sugarcane accounted for $60-70 \%$ of the total planting area [2]. Ratooning has some advantages, such as cost-reducing, labor-saving, and early ripening. However, ratooning planting faced various problems. Plantlet chlorosis is one of the problems confronting the Guangxi farmer. Approximately $40 \%$ of the sugarcane cultivated area appearing chlorosis in Guangxi, causing production reduction at about $23-40 \%[2,3]$.

Green pigment is determined by the chlorophyll content, which comprises chlorophyll a and chlorophyll b molecules [4]. In plants, leaf chlorosis is due to lacking chlorophyll. Chlorophyll metabolism involves a series of enzymes, including HemA, HemB, chlH, chIM, por, and NOL [5]. Iron deficiency triggered chlorophyll synthesis disorder, which leads to chlorosis in Areca catechu [6]. Genes related to chlorophyll metabolism were found with low expression levels in light green cucumber [7]. Chlorophyll is the primary pigment that absorbs light energy in photosynthesis reactions $[8,9]$. Low chlorophyll content in chlorosis leaf decreases the photosynthetic rate and 
fresh weight $[6,10]$. Chlorophyll biosynthesis plays a vital role in maintaining photosynthetic machinery $[11,12]$. Iron is an essential micronutrient in the synthesis of chlorophyll [13]. The lack of iron is one of the most common reasons for chlorosis [14]. However, studies suggested that even the chlorosis symptoms in Guangxi were similar to iron( $\mathrm{Fe})$ chlorosis found in India, the triggering factors remained different[15-17]. Excessive Manganese $(\mathrm{Mn})$ had been found in ratoon sugarcane chlorotic plantlets to block the uptake of active iron(Fe), which inhibited chlorophyll biosynthesis[2, 3]. Not just the content of iron or other metals, metal ion homeostasis and transportation would also impact the function of chloroplasts and photosynthesis[18]. Even in the same field, the chlorosis symptoms mainly occurred in ratoon sugarcane plantlets. The cause of chlorosis in ratoon sugarcane plantlet maybe not only the field condition. Understanding how sugarcane plantlet changes in these pathways provide a new perspective of reducing sugarcane ratoon chlorosis.

The advance of technologies in -omics enables a comprehensive approach to identify the mechanism underlying plant disease[19]. Transcriptome analysis provides a comprehensive genes expression profile of a particular phenotype, while metabolome analyzes the changes in metabolites involved in corresponding pathways $[20,21]$. In chlorophyll metabolism or other metabolisms pathways, genes interact with metabolites in a comprehensive and complicated way $[7,20]$. Comparative proteomics analysis revealed proteins involved in photosynthesis, drought-responsive, and jasmonic acid biosynthesis associated with ratoon sugarcane chlorosis symptoms [17]. Due to post-transcriptional processes, the correlation between transcriptome and proteome could be low [22]. Given this situation, exploring the mechanism under ratoon sugarcane chlorosis using transcriptomic and metabolomics analysis is still needed.

Our study aimed to depict the global response of sugarcane to ratoon chlorosis. Transcriptome and metabolome were used to explore how genes and metabolites changes correlate with chlorosis. Genes or metabolites related to chlorophyll metabolism, photosynthesis, metal ion homeostasis, and phenylopropanoid biosynthesis were analyzed. The finding of this work will provide a perspective of mechanisms of chlorosis occurring in ratoon sugarcane.

\section{Materials And Methods}

\section{Plant materials condition and chlorophyll content determination}

GuiTang49 (GT49) was the sugarcane cultivar employed in this study, which was a cross progeny of ROC 22 and Ganzhe 14. GT49 was developed by Sugarcane Research Institute, Guangxi Academy of Agricultural Sciences, China [23]. The sugarcane field was located in Fusui, Chongzuo, Guangxi, China. The sugarcanes used in this research were first planted on February 22, 2019. The canes were harvested on January 31,2021 , leaving the remained stubbles to grow. From the date ratoon sugarcane first displayed the chlorotic symptom, a survey of plantlet height, leaf number, stalk number, and tiller number was conducted every ten days. On April 18, 2021, the first expanded leaves(+1 leaves) of chlorosis (chlorosis group) and non-chlorosis (control group) plantlets were collected randomly for further experiments.

The chlorosis samples were confirmed using the SPAD chlorophyll meter (Konica Minolta, Japan) with a reading less than 10. In contrast, the planted sugarcanes with a reading higher than 40 were grouped as control samples. Approximately $0.1 \mathrm{~g}$ of leaves were rinsed and ground. The chlorophyll was extracted with $80 \%$ acetone 
for $24 \mathrm{~h}$ in dark. The content of chlorophyll was determined using the spectrophotometric method according to Arnon [24].

\section{Transcriptome analysis}

Five leaves were included in one sample. Each group contained five samples (5 replicates). The total RNA was extracted using Trizol (Invitrogen, USA)according to the manufacturer's instructions. RNA quality was assessed by Agilent 2100 Bioanalyzer (Agilent, USA) and agarose gel electrophoresis. The mRNA, enriched by Oligo(dT) beads, was fragmented and reverse transcripted into cDNA first-strand with random primers. After synthesizing second-strand cDNA, the total cDNA was purified with a QiaQuick PCR extraction kit (Qiagen, The Netherlands). Sequencing adapters were ligated to the cDNA. Size selection and PCR amplification were performed before sequencing. The sequencing was operated using Illumina HiSeq 2500 (Illumina, USA).

Reads obtained from the sequencer were filtered before assembly using fastp [25]. Due to the lack of a sugarcane reference genome, the high-quality clean reads were processed to de novo assemble using the Trinity method [26]. The completeness of assembly was assessed with BUSCO (Benchmarking Universal Single-Copy Orthologs) [27]. Gene expression level was calculated using FPKM (fragments per kilobase per million reads)method with StringTie software [28]. Analyzing by DESeq software[29], genes with fold change $\geq 2$ and $p$ value $\leq 0.05$ were considered as DEGs (differential expressed genes). GO (gene ontology) and KEGG (Kyoto encyclopedia of genes and genomes ) enrichment analysis were performed to depict the gene function and biological pathway of DEGs [30,31].

\section{Metabolome analysis}

The samples for metabolome analysis were selected for transcriptome. The leaves were ground using a mixer mill (MM 400, Retsch). 100 mg of powder was extracted overnight with $1.0 \mathrm{ml}$ of aqueous methanol containing $0.1 \mathrm{mg} / \mathrm{L}$ lidocaine as internal standard. The supernatant was obtained by centrifugation and filtration. The metabolite compounds were analyzed by LC-ESI-MS/MS system (QTRAP 6500, Sciex, USA). The chromatographic separations were performed using a Waters ACQUITY C18 column (2.1 mm * $100 \mathrm{~mm}, 18 \mu \mathrm{m}$, Waters, USA) under a flow rate of $0.4 \mathrm{ml} / \mathrm{min}$ at $40^{\circ} \mathrm{C}$. The mobile phase was water $(0.04 \%$ acetic acid) as Phase A and acidified acetonitrile (0.04\% acetic acid) as Phase B. The separation was run in a gradient condition: 95:5 Phase A/Phase B for the first 10 min, 5:95 Phase A/Phase B for the 11th to 12th min, 95:5 Phase A/Phase B for the 13th to 15th min. The effluent was detected with Sciex Triple Quad 6500 mass spectrometer (Sciex, USA) in a positive ion mode. The quantification of metabolites was performed according to multiple reaction monitoring methods [32]. Analyst 1.6.1 software was applied for data filtering, peak detection, alignment, and calculation. Metabolites were identified by searching internal and public databases (MassBank, KNApSAcK, HMDB, MoTo $D B$, and METLIN) with the $\mathrm{m} / \mathrm{z}$ values, retention times, and fragment patterns. Metabolites with thresholds of variable importance in projection $(\mathrm{VIP}) \geq 1$ and $\mathrm{p}$-value $\leq 0.05$ were considered to be significantly different between the chlorosis and control groups [33].

\section{qRT-PCR validation}

The total RNA was digested by DNase, and reverse-transcribed into cDNA for qRT-PCR using PrimeScript RT reagent kit (TAKARA, Dalian, China). The ACAD gene was used as a reference gene to normalize the relative expression level [34]. The qRT-PCR process was performed on qTOWER Real-Time Thermal Cyclers (Analytik 
Jena, Germany). The relative expression rate was calculated with the $-2 \Delta \Delta \mathrm{CT}$ method [35]. All the primers of the validation gene were listed (Table S1).

\section{Results}

Phenotype analysis of chlorosis ratoon and planted sugarcane plantlet

The ratoon plantlets would display distinct chlorosis symptoms, even in the same field, comparing with the planted cane plantlets (Figure 1A). The SPAD chlorophyll meter reading of the plantlet leaves showed that the chlorosis and planted plantlets were significantly different (Figure 1B and 1C). To monitor the growth development of the ratoon plantlet, we measured some agronomic traits. The plantlet shown chlorosis symptom grew slower than planted cane in plant height (Figure 2A), leaf (Figure 2B), stalk nod (Figure 2C), and tiller (Figure 2D).

\section{Global overview of sugarcane leaf transcriptome with chlorosis symptom}

Since the ratoon sugarcane chlorosis is displayed mainly in the leaf, we hypothesized the changes in leaf transcriptome might correlate with the symptom. Furthermore, it may correlate with rhizosphere microbiota. Using the same plantlets, we extracted the total RNA from the ten samples. A total of $621,045,632$ reads were generated using Illumina HiSeq 4000. Due to lack of reference genome, 88.339 unigenes were de novo assembled using Trinity software. The average length of all the unigenes was $903 \mathrm{bp}$. To assess De novo assembled unigenes' completeness, we took a quantitative measure with BUSCO (Benchmarking Universal Single-Copy Orthologs). The results showed the percentage of conserved orthologues of plants represented in our assembled sugarcane transcriptome. Completeness of all the unigenes resulted in a high rate of complete plant orthologues (75\%). Of the 1,440 orthologues searched in the BUSCO set of plants, 75\% was complete, with the number of 1,080. Of the 1,080 complete BUSCO orthologues, 1,060 were single-copy, while the other 20 orthologues were duplicated. The proportion of fragmented and missing BUSCOs was 14\% and 11\%, respectively (Figure $3 \mathrm{~A}$ ). To annotate and classify the assembled unigenes, we employed four public protein databases to BLASTX the deduced peptides sequence of the unigenes, resulting in 48,816 unigenes were annotated (Table S2). Of all the annotated unigenes, 48, 275 (98.9\%) unigenes showed a particular hit within the $\mathrm{Nr}$ database (Non-redundant protein sequence database). Among them, proteins were mainly from Zea mays, Setaria italica, and Oryza sativa Japonica. 5,745 unigenes only match the Nr database, while KEGG, Swissprot, and COG had 218, 176, and 35 unigenes, which were single matched, respectively (Figure 3B). A total of 17,756 unigenes were found in all four databases. All the Nr hit genes were from 479 species. Of all the hits, 17,691 (36.65\%) were significantly similar to proteins of Zea mays, and 7,406 (15.34\%) and 5,484 (11.36\%) were from Setaria italica and Oryza sativa Japonica Group, respectively (Figure 3C). According to some criteria (log2 foldchange $>1$, p-value $\leq 0.05$ ), we identified a total of 11,776 differentially expressed genes (DEG) between chlorosis and control groups (Figure 3D).

\section{Function analysis of DEGs in Chlorosis sugarcane}

Gene ontology (GO) analysis was employed to emphasize the function of DEGs. GO enrichment analysis revealed that DEGs were most enriched in GO cell component categories of membrane, thylakoid and intrinsic 
component of membrane (Figure 4A, Table S3). In molecular function categories, tetrapyrrole binding, cellulose synthase activity and catalytic activity were the most enriched terms (Figure 4B, Table S3). For the biological processes, glucan metabolic process (GO:0044042), cellular metal ion homeostasis (GO:0006875) and cellular transition metal ion homeostasis (GO:0046916) exhibited the highest association with DEGs of the chlorosis symptom (Figure4C, Table S3). To map the metabolic pathways of DEGs, we annotated these genes using KEGG analysis. A total of 31 pathways were significantly enriched from all the DEGs (Table S4). Among them, 27 pathways were related to metabolism, while two environmental information processing pathways and two organismal systems pathways were observed. Biosynthesis of secondary metabolisms, Metabolic pathways and plant-pathogen interaction were the top three enriched pathways (Figure 4D).

\section{The changes of genes related to chlorophyll metabolism in chlorosis sugarcane}

The KEGG analysis indicated that DEGs involved in the chlorophyll metabolism pathway were enriched ( $\mathrm{p}$-value $=0.0224$, Table S5). To further investigate this pathway, we found that sixteen genes were differently expressed in the chlorosis group compared to the control group, including eleven down-regulated genes and five upregulated genes (Figure 5A). The down-regulated genes encode enzymes covered almost the whole process of chlorophyll biosynthesis, including glutamyl - tRNA reductase (hemA, EC: 1.2.1.70), coproporphyrinogen III oxidase (hemF, EC: 1.3.3.3), protoporphyrinogen/coproporphyrinogen III oxidase (hemY, EC: 1.3.3.4), magnesium chelatase subunit $\mathrm{H}$ (chlH, EC: 6.6 .1 .1$), \mathrm{Mg}$ - protoporphyrin IX monomethyl ester cyclase (chIE, EC: 1.14.13.81), protochlorophyllide reductase (por, EC: 1.3.1.33) and chlorophyll b reductase (NOL, EC: 1.1.1.294). The upregulated genes encode uroporphyrinogen decarboxylase (hemE, EC: 4.1.1.37), chlorophyllase (EC: 3.1.1.14) and chlorophyll a synthase (chIG, EC: 2.5.1.62). Also, the content of chlorophyll in chlorosis samples (average 0.40 $\mathrm{mg} / \mathrm{g}$ ) was significantly lower than control samples (3.03 mg/g) (Figure 5B). The gene expression pattern in the chlorophyll metabolism pathway, in which down-regulated expression DEGs was the majority, was consistent with the reduction of chlorophyll content in chlorosis samples.

\section{The affection of photosynthesis in chlorosis ratoon sugarcane}

Chlorophyll is the primary pigment to capture light energy. Considering the lack of chlorophyll in the chlorosis group, we noticed that two photosynthesis pathways, including photosynthesis-antenna proteins and photosynthesis, were also enriched by KEGG analysis (Table S6). There were 36 DEGs related to KEGG photosynthesis pathways, including nine genes related to photosystem I (PSI), seven related to photosystem II (PSII), one related to cytochrome $\mathrm{b} 6 / \mathrm{f}$ complex, three related to photosynthetic electron transport, two related to F-type ATPase, five related to light-harvesting chlorophyll protein complex I (LHCl) and nine related to LHCII (Figure 6A and B). Additionally, 32 genes, among the 36 DEGs of photosynthesis pathways, were significantly down-regulated in the chlorosis group, while only four genes showed up-regulated expression patterns (Figure $6 C)$.

\section{Metal ion homeostasis and transport processes respond under chlorosis ratoon sugarcane}


Among the 81 enriched GO biological processes, there were four categories related to metal ion homeostasis and three categories related to metal ion transport (Figure 7A, Table S7). In the four categories related to metal ion homeostasis, including cellular metal ion homeostasis (GO: 0006875), cellular transition metal ion homeostasis (GO: 0046916), transition metal ion homeostasis (GO: 0055076) and metal ion homeostasis (GO: 0055065), there were $10 \mathrm{DEGs}$ of six down- and four up-regulated. Of the three metal ion transport processes, 31 DEGs were found with 15 down-regulated and 16 up-regulated genes (Figure 7B). Homeostasis and transport processes shared seven genes in common, which were two FER1 (unigene0002936, unigene0029675), Unidentified gene(unigene0019302), NHX2 (unigene0040569), DTX42(unigene0056614), HMA1 (unigene0064621) and HCC1 (unigene0083098). Three genes, including TMN6 (unigene0036726), YS1(unigene0055240) and IRO2(unigene0085997), were only found in processes related to metal ion homeostasis.

\section{Metabolome analysis of the changes in chlorosis sugarcane}

To disclose the differences of metabolites in ratoon chlorosis sugarcane, we performed metabolome analysis using LC-ESI-MS/MS system. A total of 498 compounds were identified and quantified (Table S8). Thirteen compounds with VIP value $\geq 1$ and $p$-value $<0.05$ were classified as significantly differentially expressed metabolites (DEMs) between the chlorosis and control groups. All these metabolites, including six phenolic acids, three lipids, one alkaloid, one organic acid and one other compound, were down-regulated in the chlorosis group (Table 1). Among them, the compound with the greatest variation in content was coniferyl alcohol with a fold change of -2.9. KEGG pathway analysis demonstrated that the phenylpropanoid biosynthesis pathway was the most and only enriched pathway among the different metabolites ( $p$-value $=0.0088$ ). Three compounds belonged to the phenylpropanoid biosynthesis, including Coniferyl alcohol, Ferulic acid and 5-OCaffeoylshikimic acid. 
Table 1

Differentially expressed metabolites between chlorosis and control groups

\begin{tabular}{|c|c|c|c|c|c|c|}
\hline Class & Compounds & Chlorosis & Control & VIP* & $\mathrm{p}$-value & $\begin{array}{l}\text { Log2_Fold } \\
\text { change }\end{array}$ \\
\hline Alkaloids & $\begin{array}{l}\text { p- } \\
\text { Hydroxymandelonitrile }\end{array}$ & 430394 & 608420 & 1.10959687 & 0.02027379 & -0.4994096 \\
\hline \multirow[t]{3}{*}{ Lipids } & $\begin{array}{l}\text { Octadeca- } \\
\text { 11E,13E,15Z-trienoic } \\
\text { acid }\end{array}$ & 3844200 & 4741460 & 2.56435704 & 0.00846175 & -0.302648 \\
\hline & Eicosadienoic acid & 461728 & 577134 & 1.01768916 & 0.00513142 & -0.3218631 \\
\hline & LysoPC 16:0 & 4335720 & 6566380 & 4.19556532 & 0.03436214 & -0.5988267 \\
\hline $\begin{array}{l}\text { Organic } \\
\text { acids }\end{array}$ & $\begin{array}{l}\text { 2-Hydroxycinnamic } \\
\text { acid }^{*}\end{array}$ & 319370 & 545734 & 1.15424233 & 0.02310167 & -0.7729691 \\
\hline Others & (S)-2-Phenyloxirane & 896878 & 1115572 & 1.19133863 & 0.02950794 & -0.3148 \\
\hline \multirow{7}{*}{$\begin{array}{l}\text { Phenolic } \\
\text { acids }\end{array}$} & Phthalic anhydride & 710886 & 857740 & 1.05578543 & 0.02138151 & -0.2709222 \\
\hline & Vanillin* & 1562940 & 1941380 & 1.53827326 & 0.00423727 & -0.3128201 \\
\hline & $\begin{array}{l}2- \\
\text { (Formylamino)benzoic } \\
\text { acid }\end{array}$ & 506076 & 742464 & 1.12174147 & 0.00254916 & -0.552967 \\
\hline & Coniferyl alcohol & 79553 & 231474 & 1.08262447 & 0.00357173 & -1.5408619 \\
\hline & Ferulic acid & 285570 & 545394 & 1.34644681 & 0.01281337 & -0.9334544 \\
\hline & Dibutyl phthalate* & 13661400 & 16021600 & 4.31621733 & 0.02367218 & -0.2299129 \\
\hline & $\begin{array}{l}\text { 5-0-Caffeoylshikimic } \\
\text { acid }\end{array}$ & 207131.2 & 406682 & 1.18067851 & 0.01341248 & -0.9733563 \\
\hline
\end{tabular}

\section{The correlation of transcriptome and metabolome in phenylopropanoid biosynthesis}

To understand the changes of chlorosis in ratoon sugarcane, we further analyzed the correlation between metabolome and transcriptome. The only enriched pathway in differentially expressed metabolites, the phenylopropanoid biosynthesis pathway, was the fifth enriched pathway in DEGs of the transcriptome. A total of 92 genes were involved with the phenylpropanoid biosynthesis pathway among the 11,776 DEGs, while three out of 13 differential metabolites belonged to this pathway. The majority of the 92 genes, which including 62 genes, were down-regulated (Figure 8, Table S9). In our transcriptome, the POD gene, encoded a peroxidase, was identified expressed by 31 DEGs with 23 down-regulated and 8 up-regulated. Also, DEGs encoded HCT, PTAL, $\mathrm{C} 3 \mathrm{H}, \mathrm{CCR}, \mathrm{F} 5 \mathrm{H}, \mathrm{BGLU}$ were mainly down-regulated in the chlorosis group. In contrast, we observed more upregulated genes in $\mathrm{CAD}$ and $4 \mathrm{CL}$. The down-regulated of the two essential genes (PTAL and $\mathrm{C} 3 \mathrm{H}$ ) could largely explain the low accumulation of the two metabolites, Ferulic acid and 5-0-Caffeoylshikimic acid (Table 1). 
Furthermore, the low accumulation of coniferyl-alcohol and down-regulation of POD and F5H indicated that the lignin biosynthesis would be impaired.

\section{qRT-PCR analysis}

To validate the DEGs expression pattern, we randomly selected 20 genes, including up-and down-regulated, high and low expression rate, for quantitative real-time (qRT)-PCR assays. The primary expression trend of the 20 genes was consistent (Figure 9A). The value of transcriptome and qRT-PCR showed a significant positive correlation with the Pearson r-value of 0.9867 . The linear regression of correlation analysis was conducted, resulting in the goodness of fit with an $\mathrm{R}$ square value of 0.9513 (Figure 9B). These results indicated that the transcriptome was reliable.

\section{Discussion}

Ratooning was an ancient, low-cost, and simple cultivation method in sugarcane production [36]. Ratoon sugarcane chlorosis is still a severe problem, caused massive loss in China [3,17]. The main reason for ratoon sugarcane chlorosis was the imbalance between Fe and Mn concentration, which may induce by acidic soil, intense leaching, and continuous cultivation [2,3]. Although causes and treatments have been studied previously, the associated regulatory mechanisms of ratoon sugarcane chlorosis still need further investigations. In this study, RNA-Seq was employed to demonstrate the global transcriptomic changes of ratoon sugarcane with chlorosis symptoms. DEGs related to chlorophyll metabolism, photosynthesis, and metal ion homeostasis were found a significant difference in expression level. In addition, combining metabolome analysis, DEGs and DEMs were enriched in the phenylopropanoid biosynthesis pathway.

In Guangxi, China, the ratoon sugarcane emerged the high possibility of chlorosis symptom that contained a low concentration of chlorophyll (Figure 1A and 5B) [3]. Chlorophyll is the primary source of pigment in plant leaves, which is correlated with the green color [4]. The well-studied chlorophyll synthesis pathway involved many essential genes $[5,37]$. HemA, which catalyzes the glutamy-tRNA to L-Glutamate 1-semialdehyde, is the initial enzyme of chlorophyll synthesis that regulates the chlorophyll accumulation during de-etiolation [38]. HemY catalyzes the protoporphyrinogen-IX to protoporphyrin-IX, which is predominant in chlorophyll synthesis [39]. The magnesium chelatases subunit $\mathrm{H}$, named $\mathrm{chlH}$, catalyzes the magnesium ion and protoporphyrin-IX to form Mg-protoporphyrin-IX, which was found induced by light [40]. Knock-down of chIE, an Mg-protoporphyrin-IX monomehyl ester cyclase, resulted in retarded growth and chloroplast developmental defects in Arabidopsis [41]. The protochlorophyllide reductase (por), the enzyme that promotes a photoreduction of protochlorophyllide to chlorophyllide, plays a vital role in the greening stage [42]. NOL, a chlorophyll b reductase, plays a role in chlorophyll b degradation [43]. In this study, twelve down-regulated DEGs, including three hemA, one hemF, two hemY, two chlH, one chIE, two por, and one NOL, were found in the chlorophyll synthesis pathway (Figure 5). Compared to the four up-regulated DEGs, including one hemF, one hemE, one chIG, and one CLH, the downregulated genes in this pathway were in the majority. The same phenomenon was observed in yellow leaf common wheat and light green cucumber $[7,44]$. These results indicated the direct relation between ratoon sugarcane chlorosis and the dysfunction of chlorophyll synthesis.

Leaf photosynthesis is positively correlated with chlorophyll content [45]. In A. thaliana, photosynthetic efficiency reduction was accompanied by a decrease in chlorophyll content [46]. In yellow leaf common wheat, 
five genes encoded proteins of the photosynthesis system were significantly down-regulated [44]. Transcriptome and proteome analysis of a wheat mutant with albino leaves shown that the expression levels of both genes and proteins related to photosynthesis were lower than wild-type green wheat [47]. Likewise, we observed similar results in this study. There were 36 DEGs found in photosynthesis pathway, of which 32 were down-regulated (Figure 6). The light-harvesting chlorophyll protein complexes (LHC) binds chlorophyll to function in the photosynthesis system [48]. Lack of the LHC would affect plants' photosynthetic rate and growth [49]. In chlorosis sugarcane, we found that all the DEGs of LHC were down-regulated (Figure 6), suggesting that the formation of photosynthesis's antenna proteins would be affected. Photosynthesis mainly occurs in the chloroplast, which is related to the green color of plants. Chloroplast comprises chloroplast membrane, thylakoid, and matrix. The multi subunits complexes, including PSI, PSII, cytochrome b6/f complex, and photosynthetic electron transport, are embedded in the thylakoid membrane [50]. DEGs of these complexes were significantly repressed in chlorosis sugarcane (Figure 6). The GO enrichment analysis of cellular components shown that five GO terms, including thylakoid, thylakoid part, photosystem, photosynthetic membrane, and plastid thylakoid, were among the top 10 enriched terms (Figure 4A). These results agreed with previous reports, which revealed the yellow leaf mutant of common wheat caused abnormal chloroplast development [51].

Studies of ratoon sugarcane chlorosis in Guangxi suggested the excessive Mn accumulation in parent stalks was the main reason for chlorosis. At the same time, the active Fe deficiency in plantlets played a secondary role [3]. The subsequent investigation revealed that, in the progression of the greening of chlorotic seedling, the leaf $\mathrm{Mn}$ content decreased significantly, whereas Fe content increased [52]. These results remind us that the metal ion metabolism may exist disorder in chlorosis sugarcane. Our GO analysis has shown that seven categories related to metal ion homeostasis and transport were significantly enriched (Figure 7A). A total of 34 DEGs were found in these categories (Figure 7B). Iron-phytosiderophore transporter (YS1) and transcription factor bHLH100 (IRO2) and were up-regulated by iron deficiency [53, 54]. Ferritin 1 (FER1), important for iron homeostasis, stores iron in a soluble form, is up-regulated by iron overload treatment[55]. In our study, two genes, YS1(unigene0055240) and IRO2(unigene0085997), shown a significantly increase with log2 FC higher than 8, while FER1 (unigene0002936, unigene0029675) were down-regulated with log2 FC lower than -6 (Table S7). These results indicated that the differential regulation of metal ion metabolism genes agreed with iron deficiency. However, Does iron deficiency induce genes differential changes? Or do the differentially expressed genes lead to iron deficiency in the leaf? This situation still needs further investigation.

In a previous study, the phenylpropanoid biosynthesis pathway was enriched in both transcriptome and metabolome analysis of chlorosis $A$. catechu L. [6]. Combining metabolome analysis, we found 62 DEGs and 3 DEMs (differentially expressed metabolites) belong to the phenylpropanoid biosynthesis pathway (Figure 8, Table S9, and Table 1). Interestingly, phenylpropanoid biosynthesis was the only enriched pathway in DEMs. These results indicated that phenylpropanoid biosynthesis is involved in ratoon sugarcane chlorosis development. Phenylpropanoids contribute significantly to plants' response towards biotic and abiotic stimuli [56]. In chlorotic tea leaves, genes and metabolites related to phenylpropanoids biosynthesis showed lower expression levels than green leaf [57]. In an iron deficiency chlorosis tolerant soybean, genes in the phenylpropanoid biosynthesis were up-regulated in low Fe condition [58]. Synthesis of coumarins is part of Fe acquisition machinery in Arabidopsis [59]. We noticed the BGLU, a beta-glucosidase coding gene, was downregulated in chlorosis sugarcane (Figure 8 ). This result suggests that the phenylpropanoid biosynthesis was altered at the transcripts and metabolites level in chlorotic ratoon sugarcane. Given the central role of

Page $10 / 23$ 
phenylpropanoid biosynthesis in metabolites pool $[56,60]$, two secondary metabolites pathways, including flavonoids and phenylalanine, were found enriched in KEGG analysis (Figure 4D, Table S4).

\section{Conclusions}

This study has investigated the changes in transcriptome and metabolome between chlorotic and non-chlorotic sugarcane leaves. Our results provided an insight into chlorophyll synthesis, photosynthesis, metal ion metabolism, and phenylpropanoids biosynthesis in ratoon chlorosis sugarcane. Genes regulation dysfunction in metal ion homeostasis and transportation likely leads to Mn toxicity and Fe deficiency, which may cause ratoon chlorosis in sugarcane [3]. The reduction of chlorophyll content is likely due to the global gene expression decrease of the chlorophyll synthesis pathway. Furthermore, the photosynthesis pathway was affected significantly. The consistent results of metabolome and transcriptome in phenylpropanoids biosynthesis pathway suggests that the biosynthesis of secondary metabolites plays a vital role in ratoon sugarcane chlorosis.

\section{Declarations}

Conflict of Interest

The authors declared that this study was conducted in the absence of any commercial or financial relationships that could be construed as a potential conflict of interest.

\section{Author Contributions}

TL, ZZ, DY, YF, LQ, RC, HY, HZ, and JW conceived and designed the experiments. TL, ZZ, and DY collected the samples. TL, YF, LQ, and QC performed the transcriptome sequencing and data analysis. TL, RC, $H Y$, and $H Z$ contributed equally to the metabolome analysis. TL wrote the paper. JW supervised this project.

Funding

This research was supported by grants from National Natural Science Foundation of China (No. 32001484), Chines Academy of Science and Technology Service Network Planning (No. KFJ-STS-QYZD-199-2 ), Natural Science Foundation of Guangxi Zhuang Autonomous Region (No. 2019GXNSFBA245005), and Innovation Team Project for Modern Agricultural Industrial Technology System of Guangxi (No. nycytxgxcxtd-2021-03).

Availablity of data and materials

The raw reads generated in this study have been deposited in BioProject with the accession number of PRJNA787323 (https://www.ncbi.nlm.nih.gov/bioproject/PRJNA787323).

\section{References}

1. Zhang M, Govindaraju M: Sugarcane production in China. Sugarcane-technology and research 2018, 49. 
2. Chen G-F, Liu Z, Huang Y-Y, Xiong L-M, Tan Y-M, Xing Y, Tang Q-Z: Factors responsible for sugarcane ratoon chlorosis in acid soil and its management in Guangxi province of China. Sugar Tech 2016, 18(5):500504.

3. Huang YL, Yang S, Long GX, Zhao ZK, Li XF, Gu MH: Manganese Toxicity in Sugarcane Plantlets Grown on Acidic Soils of Southern China. PLOS ONE 2016, 11(3):e0148956.

4. da Silva Ferreira V, Sant'Anna C: Impact of culture conditions on the chlorophyll content of microalgae for biotechnological applications. World Journal of Microbiology and Biotechnology 2016, 33(1):20.

5. Tanaka A, Tanaka R: Chlorophyll metabolism. Curr Opin Plant Biol 2006, 9(3):248-255.

6. Li J, Cao X, Jia X, Liu L, Cao H, Qin W, Li M: Iron Deficiency Leads to Chlorosis Through Impacting Chlorophyll Synthesis and Nitrogen Metabolism in Areca catechu L. Frontiers in Plant Science 2021, 12(1577).

7. Wang M, Chen L, Liang Z, He X, Liu W, Jiang B, Yan J, Sun P, Cao Z, Peng Q et al: Metabolome and transcriptome analyses reveal chlorophyll and anthocyanin metabolism pathway associated with cucumber fruit skin color. BMC Plant Biology 2020, 20(1):386.

8. Mandal R, Dutta G: From photosynthesis to biosensing: Chlorophyll proves to be a versatile molecule. Sensors International 2020, 1:100058.

9. $\quad$ Li Y, He N, Hou J, Xu L, Liu C, Zhang J, Wang Q, Zhang X, Wu X: Factors Influencing Leaf Chlorophyll Content in Natural Forests at the Biome Scale. Frontiers in Ecology and Evolution 2018, 6(64).

10. Zhou K, Ren Y, Lv J, Wang Y, Liu F, Zhou F, Zhao S, Chen S, Peng C, Zhang X et al: Young Leaf Chlorosis 1, a chloroplast-localized gene required for chlorophyll and lutein accumulation during early leaf development in rice. Planta 2013, 237(1):279-292.

11. Liu L, Lin N, Liu X, Yang S, Wang W, Wan X: From Chloroplast Biogenesis to Chlorophyll Accumulation: The Interplay of Light and Hormones on Gene Expression in Camellia sinensis cv. Shuchazao Leaves. Frontiers in Plant Science 2020, 11(256).

12. Islam S, Bhor SA, Tanaka K, Sakamoto H, Yaeno T, Kaya H, Kobayashi K: Transcriptome Analysis Shows Activation of Stress and Defense Responses by Silencing of Chlorophyll Biosynthetic Enzyme CHLI in Transgenic Tobacco. International Journal of Molecular Sciences 2020, 21(19):7044.

13. Moran Lauter AN, Peiffer GA, Yin T, Whitham SA, Cook D, Shoemaker RC, Graham MA: Identification of candidate genes involved in early iron deficiency chlorosis signaling in soybean (Glycine max) roots and leaves. BMC Genomics 2014, 15(1):702.

14. Lucena JJ: Effects of bicarbonate, nitrate and other environmental factors on iron deficiency chlorosis. A review. Journal of Plant Nutrition 2000, 23(11-12):1591-1606.

15. Shrivastava A, Srivastava M, Shukla S, Gaur A, Darash R: Chlorosis-induced alterations in leaf laminae of sugarcane. In: 2004. 
16. Radhamani R, Kannan R, Rakkiyappan P: Leaf Chlorophyll Meter Readings as an Indicator for Sugarcane Yield Under Iron Deficient Typic Haplustert. Sugar Tech 2016, 18(1):61-66.

17. Fan Y-G, Chen R-F, Qiu L-H, Zhou Z-F, Zhou H-W, Wei J-G, Song X-P, Luo T, Lakshmanan P, Huang X et al: Quantitative Proteomics Analysis of Sugarcane Ratoon Crop Chlorosis. Sugar Tech 2021, 23(3):673-681.

18. Yruela I: Transition metals in plant photosynthesis. Metallomics 2013, 5(9):1090-1109.

19. Crandall SG, Gold KM, Jiménez-Gasco MDM, Filgueiras CC, Willett DS: A multi-omics approach to solving problems in plant disease ecology. PloS one 2020, 15(9):e0237975-e0237975.

20. Li Y, Fang J, Qi X, Lin M, Zhong Y, Sun L, Cui W: Combined Analysis of the Fruit Metabolome and Transcriptome Reveals Candidate Genes Involved in Flavonoid Biosynthesis in Actinidia arguta. International journal of molecular sciences 2018, 19(5):1471.

21. Wang Z, Cui Y, Vainstein A, Chen S, Ma H: Regulation of fig (Ficus carica L.) fruit color: metabolomic and transcriptomic analyses of the flavonoid biosynthetic pathway. Frontiers in Plant Science 2017, 8:1990.

22. Bathke J, Konzer A, Remes B, Mclntosh M, Klug G: Comparative analyses of the variation of the transcriptome and proteome of Rhodobacter sphaeroides throughout growth. BMC Genomics 2019, 20(1):358.

23. Elsheery NI, Sunoj VSJ, Wen Y, Zhu JJ, Muralidharan G, Cao KF: Foliar application of nanoparticles mitigates the chilling effect on photosynthesis and photoprotection in sugarcane. Plant Physiology and Biochemistry 2020, 149:50-60.

24. Arnon DI: COPPER ENZYMES IN ISOLATED CHLOROPLASTS. POLYPHENOLOXIDASE IN BETA VULGARIS. Plant Physiol 1949, 24(1):1-15.

25. Chen S, Zhou Y, Chen Y, Gu J: fastp: an ultra-fast all-in-one FASTQ preprocessor. Bioinformatics 2018, 34(17):i884-i890.

26. Grabherr MG, Haas BJ, Yassour M, Levin JZ, Thompson DA, Amit I, Adiconis X, Fan L, Raychowdhury R, Zeng Q et al: Full-length transcriptome assembly from RNA-Seq data without a reference genome. Nature Biotechnology 2011, 29(7):644-652.

27. Simão FA, Waterhouse RM, loannidis P, Kriventseva EV, Zdobnov EM: BUSCO: assessing genome assembly and annotation completeness with single-copy orthologs. Bioinformatics 2015, 31(19):3210-3212.

28. Pertea M, Kim D, Pertea GM, Leek JT, Salzberg SL: Transcript-level expression analysis of RNA-seq experiments with HISAT, StringTie and Ballgown. Nat Protoc 2016, 11(9):1650-1667.

29. Love MI, Huber W, Anders S: Moderated estimation of fold change and dispersion for RNA-seq data with DESeq2. Genome Biology 2014, 15(12):550.

30. Ashburner M, Ball CA, Blake JA, Botstein D, Butler H, Cherry JM, Davis AP, Dolinski K, Dwight SS, Eppig JT et al: Gene Ontology: tool for the unification of biology. Nature Genetics 2000, 25(1):25-29. 
31. Kanehisa M, Goto S: KEGG: kyoto encyclopedia of genes and genomes. Nucleic Acids Res 2000, 28(1):27-30.

32. Chen W, Gong L, Guo Z, Wang W, Zhang H, Liu X, Yu S, Xiong L, Luo J: A Novel Integrated Method for Large-Scale Detection, Identification, and Quantification of Widely Targeted Metabolites: Application in the Study of Rice Metabolomics. Molecular Plant 2013, 6(6):1769-1780.

33. Yun Y-H, Liang F, Deng B-C, Lai G-B, Gonçalves CMV, Lu H-M, Yan J, Huang X, Yi L-Z, Liang Y-Z: Informative metabolites identification by variable importance analysis based on random variable combination. Metabolomics 2015, 11(6):1539-1551.

34. Huang N, Ling H, Liu F, Su Y, Su W, Mao H, Zhang X, Wang L, Chen R, Que Y: Identification and evaluation of PCR reference genes for host and pathogen in sugarcane-Sporisorium scitamineum interaction system. BMC Genomics 2018, 19(1):479.

35. Livak KJ, Schmittgen TD: Analysis of relative gene expression data using real-time quantitative PCR and the 2- $\Delta \Delta C T$ method. methods 2001, 25(4):402-408.

36. Zhou MM, Shoko MD: Simultaneous selection for yield and ratooning ability in sugarcane genotypes using analysis of covariance. South African Journal of Plant and Soil 2012, 29(2):93-100.

37. Lai B, Hu B, Qin Y-H, Zhao J-T, Wang H-C, Hu G-B: Transcriptomic analysis of Litchi chinensis pericarp during maturation with a focus on chlorophyll degradation and flavonoid biosynthesis. BMC genomics 2015, 16(1):225-225.

38. McCormac AC, Fischer A, Kumar AM, Söll D, Terry MJ: Regulation of HEMA1 expression by phytochrome and a plastid signal during deetiolation in Arabidopsis thaliana. Plant J 2001, 25(5):549-561.

39. Lermontova I, Kruse E, Mock HP, Grimm B: Cloning and characterization of a plastidal and a mitochondrial isoform of tobacco protoporphyrinogen IX oxidase. Proc Natl Acad Sci U S A 1997, 94(16):88958900.

40. Stephenson PG, Terry MJ: Light signalling pathways regulating the Mg-chelatase branchpoint of chlorophyll synthesis during deetiolation in Arabidopsis thaliana. Photochem Photobiol Sci 2008, 7(10):12431252.

41. Bang WY, Jeong IS, Kim DW, Im CH, Ji C, Hwang SM, Kim SW, Son YS, Jeong J, Shiina T et al: Role of Arabidopsis $\mathrm{CHL} 27$ protein for photosynthesis, chloroplast development and gene expression profiling. Plant Cell Physiol 2008, 49(9):1350-1363.

42. Armstrong GA, Runge S, Frick G, Sperling U, Apel K: Identification of NADPH:protochlorophyllide oxidoreductases A and B: a branched pathway for light-dependent chlorophyll biosynthesis in Arabidopsis thaliana. Plant Physiol 1995, 108(4):1505-1517.

43. Horie $\mathrm{Y}$, Ito $\mathrm{H}$, Kusaba M, Tanaka R, Tanaka A: Participation of chlorophyll b reductase in the initial step of the degradation of light-harvesting chlorophyll a/b-protein complexes in Arabidopsis. J Biol Chem 2009, 
44. Wu H, Shi N, An X, Liu C, Fu H, Cao L, Feng Y, Sun D, Zhang L: Candidate Genes for Yellow Leaf Color in Common Wheat (Triticum aestivum L.) and Major Related Metabolic Pathways according to Transcriptome Profiling. International Journal of Molecular Sciences 2018, 19(6):1594.

45. Buntin GD, Braman SK, Gilbertz DA, Phillips DV: Chlorosis, Photosynthesis, and Transpiration of Azalea Leaves After Azalea Lace Bug (Heteroptera: Tingidae) Feeding Injury. Journal of Economic Entomology 1996, 89(4):990-995.

46. Plötner B, Nurmi M, Fischer A, Watanabe M, Schneeberger K, Holm S, Vaid N, Schöttler MA, Walther D, Hoefgen $\mathrm{R}$ et al: Chlorosis caused by two recessively interacting genes reveals a role of RNA helicase in hybrid breakdown in Arabidopsis thaliana. Plant J 2017, 91(2):251-262.

47. Shi K, Gu J, Guo H, Zhao L, Xie Y, Xiong H, Li J, Zhao S, Song X, Liu L: Transcriptome and proteomic analyses reveal multiple differences associated with chloroplast development in the spaceflight-induced wheat albino mutant mta. PLOS ONE 2017, 12(5):e0177992.

48. Hoffman GE, Sanchez-Puerta MV, Delwiche CF: Evolution of light-harvesting complex proteins from Chl c-containing algae. BMC Evolutionary Biology 2011, 11(1):101.

49. Kovács Ls, Damkjær J, Kereïche S, Ilioaia C, Ruban AV, Boekema EJ, Jansson S, Horton P: Lack of the Light-Harvesting Complex CP24 Affects the Structure and Function of the Grana Membranes of Higher Plant Chloroplasts. The Plant Cell 2006, 18(11):3106-3120.

50. Allen JF, de Paula WB, Puthiyaveetil S, Nield J: A structural phylogenetic map for chloroplast photosynthesis. Trends in plant science 2011, 16(12):645-655.

51. Zhang L, Liu C, An X, Wu H, Feng Y, Wang H, Sun D: Identification and genetic mapping of a novel incompletely dominant yellow leaf color gene, Y1718, on chromosome 2BS in wheat. Euphytica 2017, 213(7):141.

52. Ling GZ, Wang XX, Yang S, Tang XL, Jia SJ, Chang MM, Li XF: Natural Amelioration of Mn-induced Chlorosis Facilitated by Mn Down-regulation, Ammonium and Rainwater in Sugarcane Seedlings. bioRxiv 2019:618124.

53. Wang HY, Klatte M, Jakoby M, Bäumlein H, Weisshaar B, Bauer P: Iron deficiency-mediated stress regulation of four subgroup Ib BHLH genes in Arabidopsis thaliana. Planta 2007, 226(4):897-908.

54. Curie C, Panaviene Z, Loulergue C, Dellaporta SL, Briat JF, Walker EL: Maize yellow stripe1 encodes a membrane protein directly involved in Fe(III) uptake. Nature 2001, 409(6818):346-349.

55. Petit JM, Briat JF, Lobréaux S: Structure and differential expression of the four members of the Arabidopsis thaliana ferritin gene family. Biochem J 2001, 359(Pt 3):575-582.

56. Vogt T: Phenylpropanoid Biosynthesis. Molecular Plant 2010, 3(1):2-20. 
57. Zhang Q, Liu M, Ruan J: Metabolomics analysis reveals the metabolic and functional roles of flavonoids in light-sensitive tea leaves. BMC Plant Biology 2017, 17(1):64.

58. Waters BM, Amundsen K, Graef G: Gene Expression Profiling of Iron Deficiency Chlorosis Sensitive and Tolerant Soybean Indicates Key Roles for Phenylpropanoids under Alkalinity Stress. Frontiers in Plant Science 2018, 9(10).

59. Schmid NB, Giehl RFH, Döll S, Mock H-P, Strehmel N, Scheel D, Kong X, Hider RC, von Wirén N: FeruloylCoA 6'-Hydroxylase1-Dependent Coumarins Mediate Iron Acquisition from Alkaline Substrates in Arabidopsis Plant Physiol 2013, 164(1):160-172.

60. Fraser CM, Chapple C: The phenylpropanoid pathway in Arabidopsis. Arabidopsis Book 2011, 9:e0152e0152.

Figures 
A

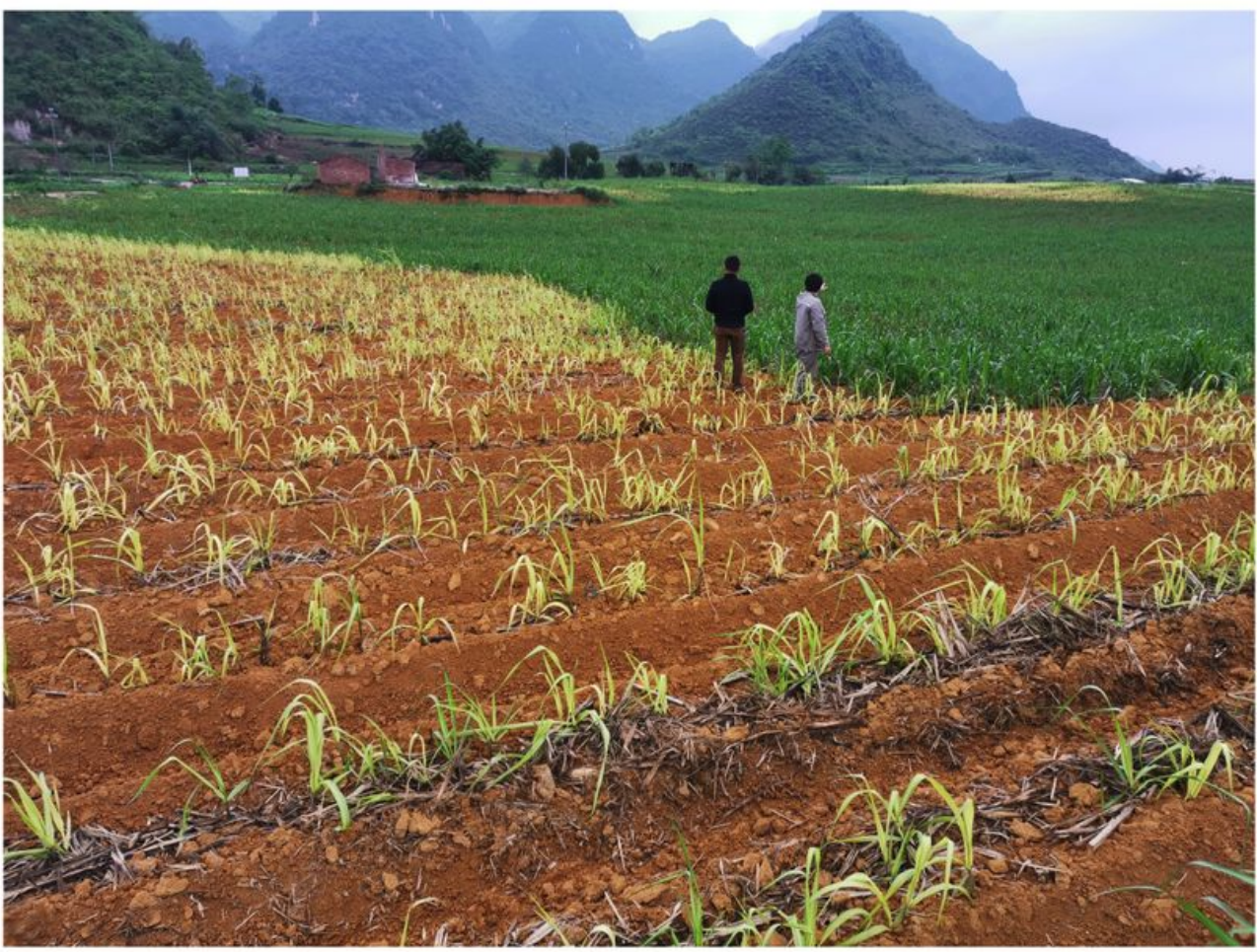

B

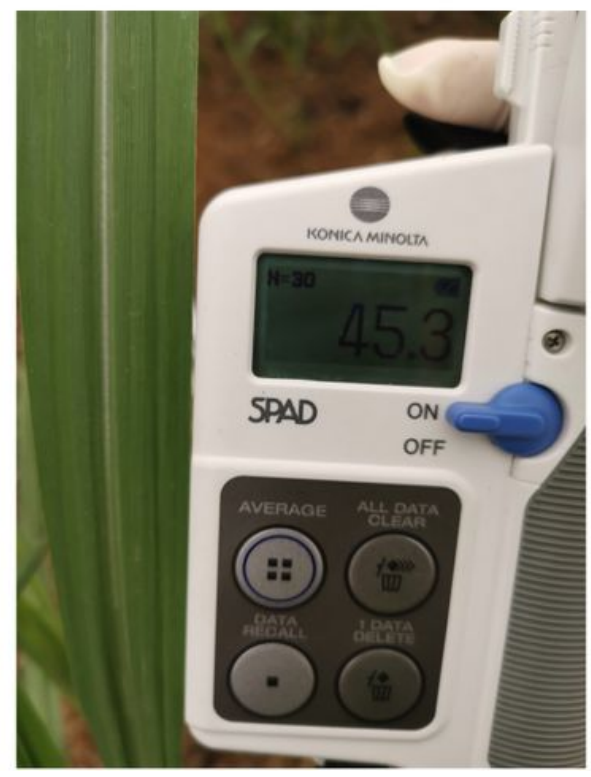

C

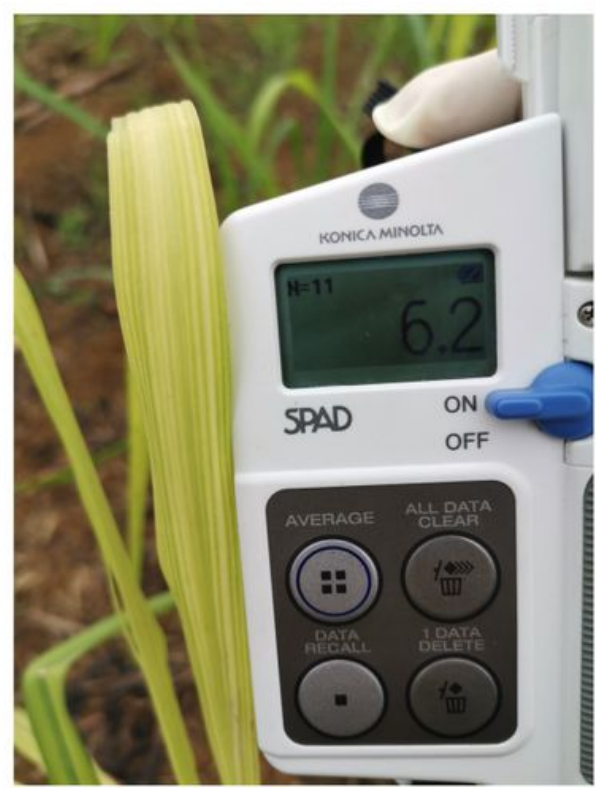

\section{Figure 1}

The sugarcane field with planted green plantlet and ratoon chlorotic plantlet (A). The green leave of control group (B) was collected with SPAD meter read higher than 40. The chlorotic leave of chlorosis group (C) was collected when the SPAD reads lower than 15. 
A

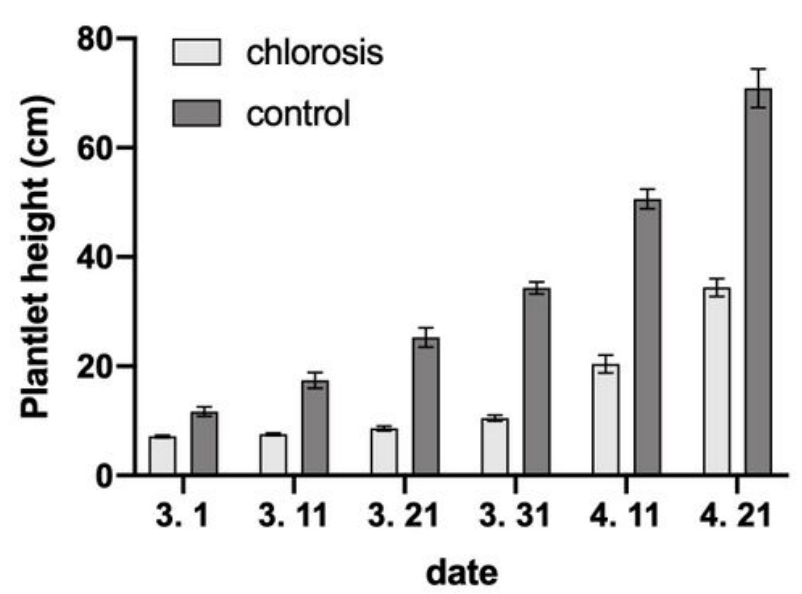

C

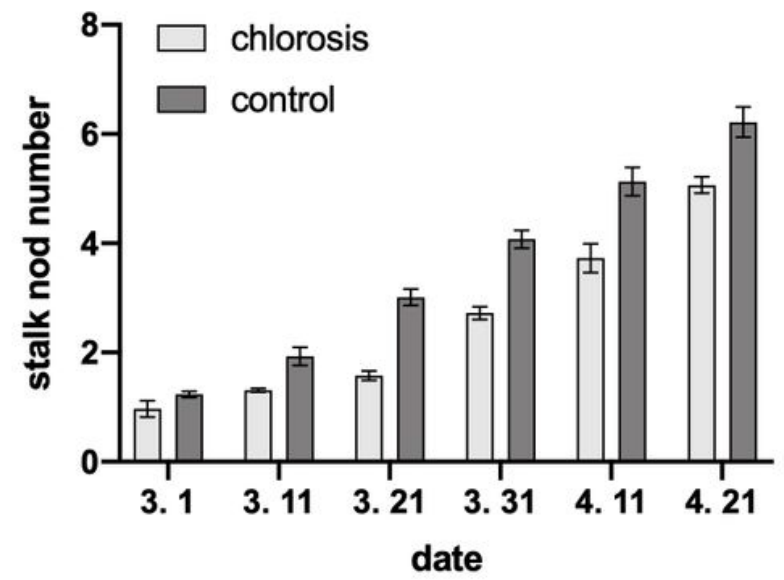

B

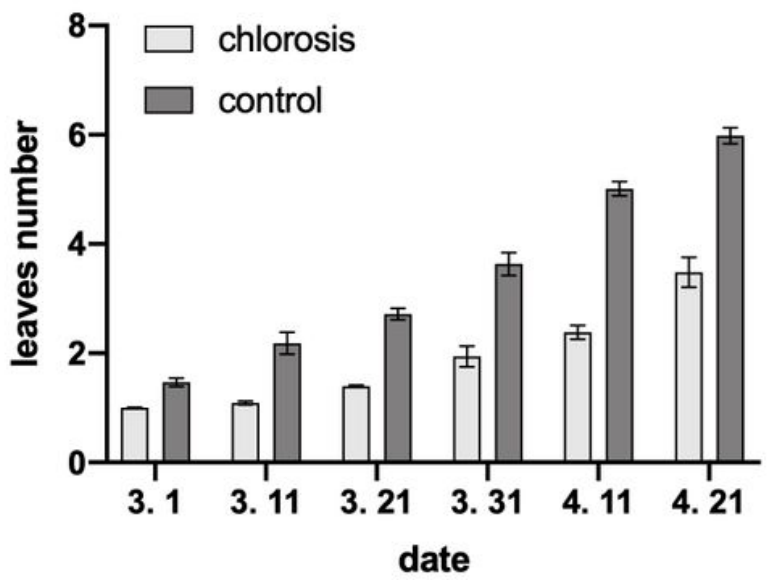

D

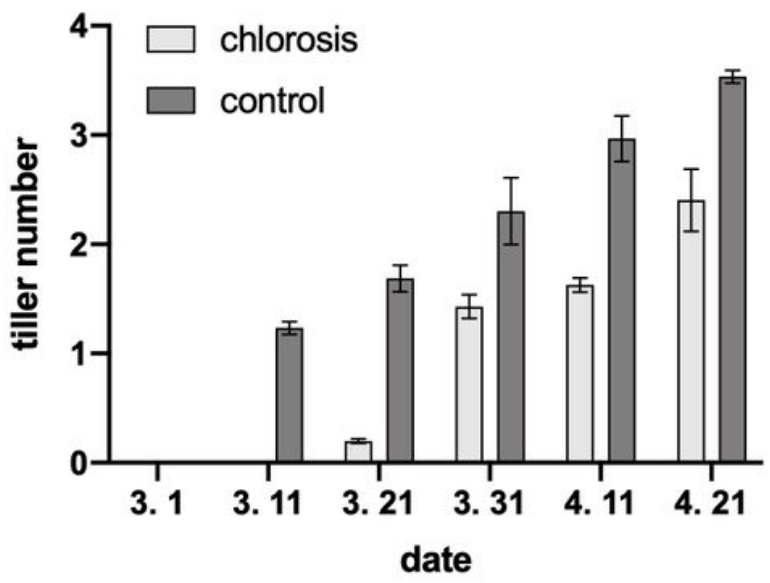

Figure 2

The plantlet agronomic traits measurement in plant height (A), leaf number (B), stalk nod number (C), and tiller number (D). The $x$ axis represents the date of sampling. The y axis represents the measurement of each agronomic trait.

\section{Figure 3}

Summary of transcriptome of sugarcane in ratoon chlorosis leaves and planted plantlet green leaves.

Transcriptome de novo assembly completeness analysis using BUSCO alignment (A). Complete orthologues include single copy (blue) and duplicated copies (green). Incomplete orthologues are fragmented (yellow). The missing (red) copies are not found in BUSCO database. The venn diagram of aligned and annotated assembly using multi databases (B). The distribution of species annotation unigenes (C). The volcano plot of expression unigenes (D). The up- and down- regulated genes are represent as red and yellow dots, while the light blue dots indicate the unigenes without significant changes. The unigenes with fold change higher than 2 and $p$-value lower than 0.05 are determined as DEGs. 


\section{Figure 4}

GO and KEGG pathway enrichment analysis of DEGs. The 10 most enriched GO terms in cellular component (A), molecular function (B) and biological process (C). The number of $X$ axial represents the ratio of DEGs in each term. Circle size denotes gene number. The 10 most enriched KEGG pathways (D). The number near each column represent the gene number and percent of that pathway, respectively. High and low p-values are denoted in red and blue, respectively.

\section{Figure 5}

The diagram of chlorophyll metabolism pathway. (A) The chlorophyll metabolism pathway is presented in a way of successive reaction steps. The circle denotes chemical compound. The gene name upon arrow denotes RNA or protein. The normalized gene expression is showed in box with colors. The direction of arrow means activation. Colour gradients from green to red represents the Log2FC of the genes. (B) The chlorophyll concentrations in sugarcane leaves of control and chlorosis samples. Each value represents a sample. The red line indicates the mean value of each group. Unpaired $t$ test was used to compare the different between the two groups. $\mathrm{p}$-value $=0.024$. ${ }^{* \star}$ denotes highly significant. hemA, glutamyl-tRNA reductase; hemL, glutamate-1semialdehyde 2,1-aminomutase; hemB, porphobilinogen synthase; hemE, uroporphyrinogen decarboxylase; hemF, coproporphyrinogen III oxidase; hemY, protoporphyrinogen/coproporphyrinogen III oxidase; chlH, magnesium chelatase subunit $\mathrm{H}$; bchM, magnesium-protoporphyrin 0-methyltransferase; chlE, magnesiumprotoporphyrin IX monomethyl ester (oxidative) cyclase; por, protochlorophyllide reductase; DVR, divinyl chlorophyllide a 8-vinyl-reductase; chIG, chlorophyll/bacteriochlorophyll a synthase; CLH, chlorophyllase; HCAR, 7-hydroxymethyl chlorophyll a reductase; NOL, chlorophyll(ide) b reductase; CAO, chlorophyllide a oxygenase. 
A
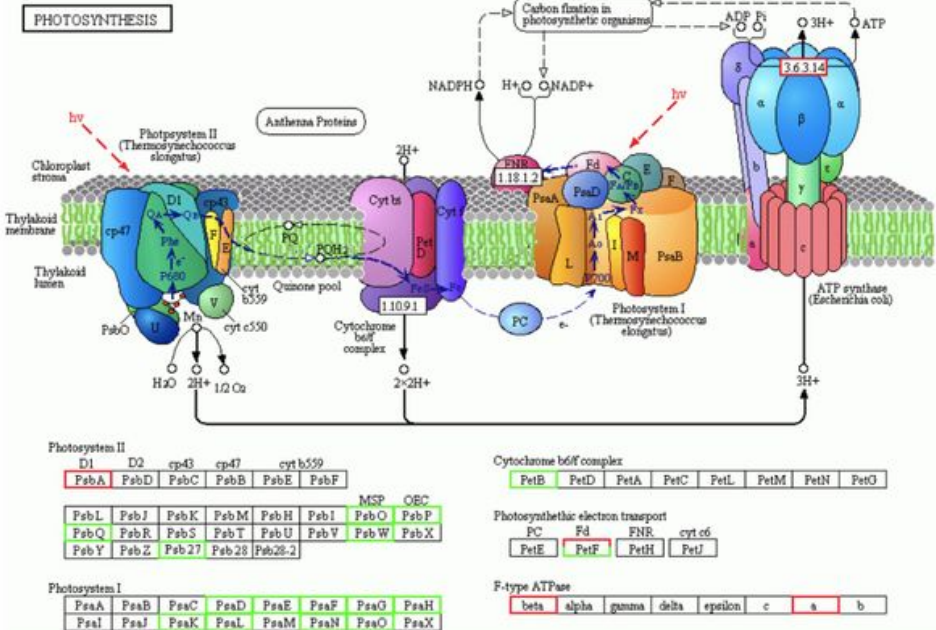

B

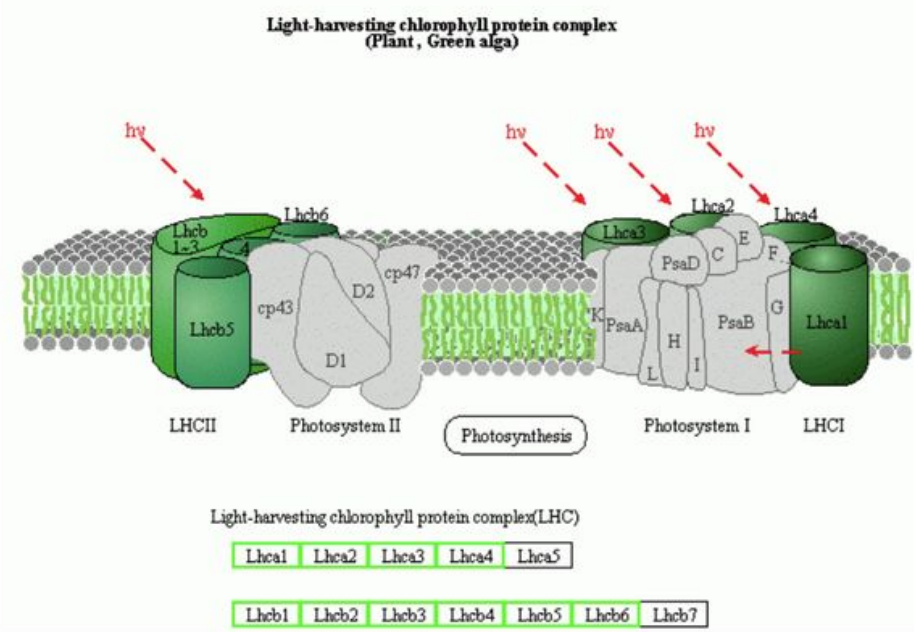

C

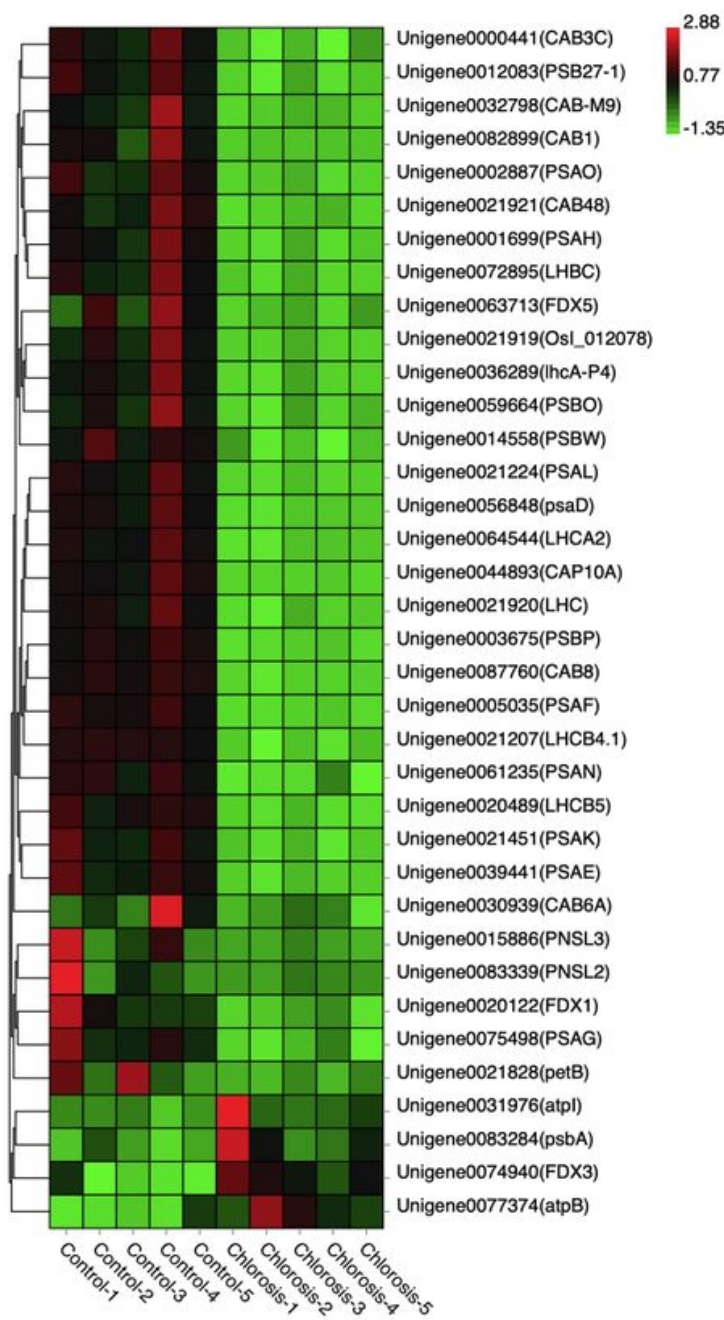

Figure 6

DEGs related to the photosynthesis pathways. (A) The KEGG pathway map of photosynthesis. (B) The KEGG pathway map of photosynthesis-antenna protein. The images were obtained from KEGG database. The DEGs expression pattern were used to annotated and generated a corresponding map. The green box with gene symbols denotes down-regulated expression in chlorosis group, while the red box denotes up-regulated expression. The genes without significant change were displayed with grey box. (C) Expression profile of genes related to photosynthesis pathways. The vertical column represents a sample. The horizontal row represents a gene. The expression ratios are based on log2 RPKM value, and normalize at row level. Each gene is presented with gene ID and gene name. PsbA-Psb27, photosystem II structure proteins; PsaA-PsaX, photosystem I structure proteins; PetB-PetG, cytochrome b6/f complex proteins; PetF, ferredoxin; beta, fF-type $\mathrm{H}+/ \mathrm{Na}+-$ transporting ATPase subunit beta; a, F-type H+-transporting ATPase subunit a; Lhca1-Lhca5, light-harvesting complex I chlorophyll a/b binding protein; Lhcb1-Lhcb7, light-harvesting complex II chlorophyll a/b binding protein 1. 
A

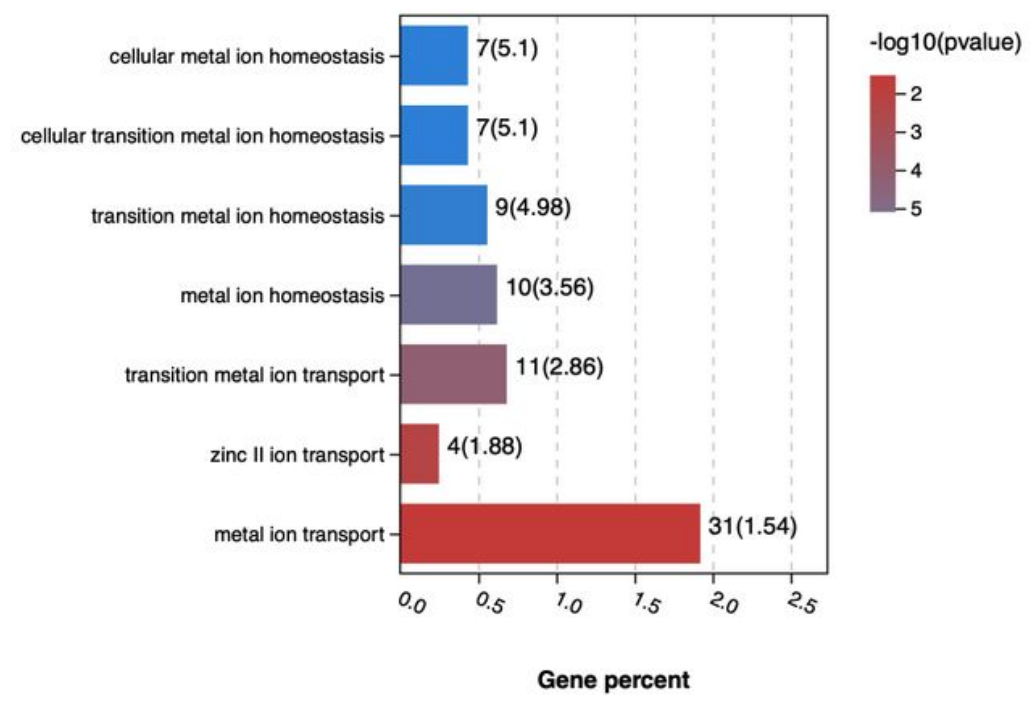

B

DEGs related to metal ion homeostasis and transport

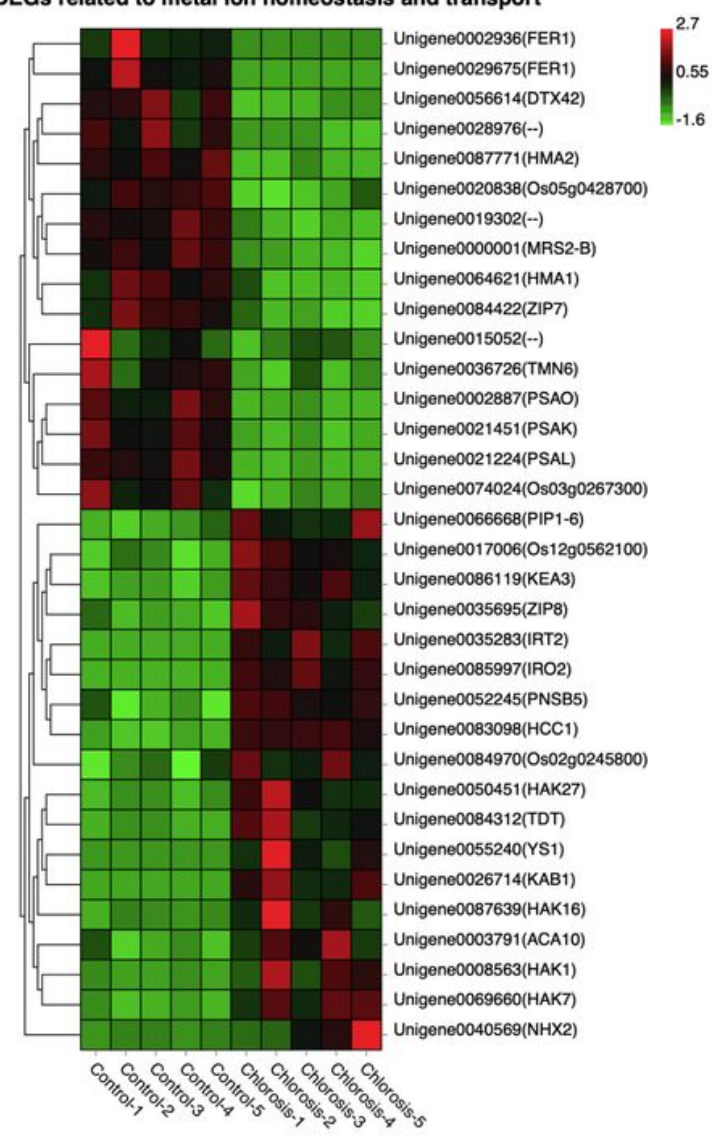

Figure 7

KEGG enrichment analysis and expression profiles of DEGs related to metal ion homeostasis or transport. (A) The seven enriched pathways related to metal ion homeostasis or transport ( $p$-value < 0.05). The number near each column represent the gene number and percent of that pathway, respectively. High and low p-values are denoted in red and blue, respectively. (B) Heat map of expression profile of genes related to metal ion homeostasis or transport. 


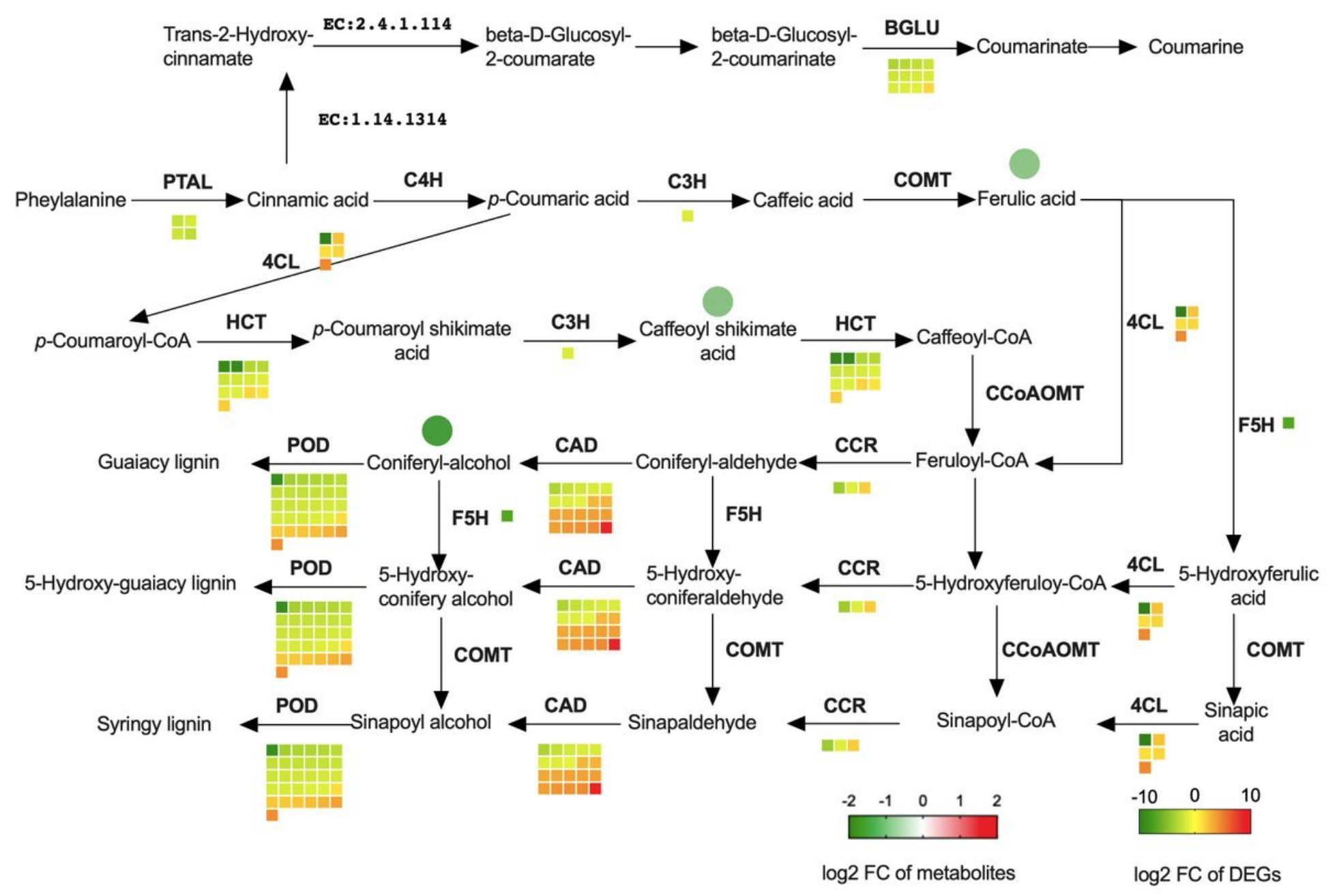

\section{Figure 8}

The diagram of phenylpropanoid synthesis pathway. The names in light-type letter are metabolites compounds. The DEGs are exhibited in bold-type letter upon the arrow. The fold change of DEGs and metabolites is shown in heatmap style. Square denotes DEGs, while the circle denotes the DEMs. Colour gradients from green to red represents the Log2FC of the genes or metabolites. PTAL, phenylalanine/tyrosine ammonia-lyase; $\mathrm{C} 4 \mathrm{H}$, trans-

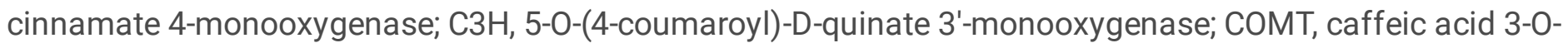
methyltransferase $4 \mathrm{CL}$, 4-coumarate-CoA ligase; HCT, shikimate O-hydroxycinnamoyltransferase; CCoAOMT, caffeoyl-CoA 0-methyltransferase; CCR, cinnamoyl-CoA reductase; CAD, cinnamyl-alcohol dehydrogenase; POD, peroxidase; F5H, ferulate-5-hydroxylase; EC:1.14.13.14, trans-cinnamate 2-monooxygenase; EC:2.4.1.114, 2coumarate O-beta-glucosyltransferase; BGLU, beta-glucosidase.

\section{Figure 9}

The validation of transcriptome using qRT-PCR. (A) The 20 genes expression pattern of transcriptome and qRTPCR. The columns in black and grey denote the expression value of transcriptome and qRT-PCR, respectively. The value represents the log2 fold change in the chlorosis group comparing with the control group. (B) Correlation of transcriptome (x-axis) and qRT-PCR (y-axis) data. 


\section{Supplementary Files}

This is a list of supplementary files associated with this preprint. Click to download.

- TableS1primersofvalidation.xls

- Tables2allgenesexpressionandannotation.xls

- TableS3GOenrichment.xls

- TableS4KEGGenrichment.xls

- TableS5DEGsofchlorophyllmetabolism.xls

- TableS6DEGsofphotosynthesispathways.xls

- TableS7DEGsofmetaliontransport.xls

- Tables8allmetabolites.xls

- TableS9DEGsofphenylopropanoidbiosynthesis.xls 